\title{
THE EFFECT OF NEUTRONS ON THE CHARACTERISTICS \\ OF THE INSULATED GATE BIPOLAR TRANSISTOR (IGBT)*
}

\author{
A. R. Hefner ${ }^{\dagger \ddagger}$, D. L. Blackburn ${ }^{\dagger}$, and K. F. Galloway ${ }^{\dagger \ddagger}$
}

\begin{abstract}
The effects of neutrons on the operating characteristics of Insulated Gate Bipolar Transistors (IGBT) are described. Experimental results are presented for devices that have been irradiated up to a fluence of $10^{13}$ neutrons $/ \mathrm{cm}^{2}$, and an analytical model is presented which explains the observed effects. It is found that the on-state voltage increases, the switching time decreases, and the saturation current decreases with increasing neutron fluence. For the range of fluences studied, the observed effects result from a reduction in minority carrier lifetime in the IGBT and not from changes in the effective dopant density. The effects of neutrons on the IGBT are compared with the known effects on power MOSFETs, and it is shown that the IGBT characteristics begin to degrade at a fluence that is an order of magnitude less than the fluence at which the power MOSFET begins to degrade.
\end{abstract}

\section{Introduction}

Recently, a new power semiconductor device has been introduced which is designed to overcome the high on-state resistance of the power MOSFET while maintaining the simple gate drive requirements of that device. ${ }^{1,2}$ The new device is controlled by a voltage at the input, such as for a MOSFET, but the output is characteristic of a bipolar transistor, hence the name Insulated Gate Bipolar Transistor (IGBT).

There have been numerous studies of the effects of radiation on power MOSFETs. ${ }^{3-12}$ Studies of radiation effects on IGBTs have been primarily for processing purposes, with the goal of optimizing the device electrical characteristics. ${ }^{13-15}$ Although no studies of the effects of ionizing radiation on IGBT characteristics have been reported, it is expected that the total dose effects should be very similar to those for power MOSFETs. That is, both the threshold voltage and anode-cathode breakdown voltage (analogous to the MOSFET drain-source breakdown voltage) should shift due to oxide- and interfacetrapped charge resulting from total dose effects. ${ }^{3-11}$ It has also been shown that a high dose-rate (of ionizing radiation) can cause the power MOSFET to burn out, probably due to activation of a parasitic bipolar transistor structure in the power MOSFET. ${ }^{12}$ It is unclear if the IGBT experiences a similar effect in a high dose-rate environment (possibly due to latch-up of a parasitic thyristor structure in the IGBT).

The primary effects of neutrons on the characteristics of IGBTs should be different from their effects on the characteristics of power MOSFETs. It is well known that exposure to neutrons causes an increase in the effective resistivity and a decrease in the carrier lifetime of silicon. The normal operating characteristics of the power MOSFET are not sensitive to changes in carrier lifetime, but they are very sensitive to changes in resistivity of the drain region material. Exposure to neutrons causes the on-resistance of power MOSFETs to increase. ${ }^{5}$ The operating characteristics of the IGBT are very sensitive to both carrier lifetime and material resistivity. This is because the output characteristics of the IGBT are those of a bipolar transistor which are sensitive to changes in both lifetime and resistivity. In analogy to what has been observed

\footnotetext{
* Supported in part by the Defense Nuclear Agency through the Naval Weapons Support Center.

$\dagger$ Semiconductor Electronics Division, National Bureau of Standards, Gaithersburg, MD 20899

‡ Electrical Engineering Department, University of Maryland, College Park, MD 20742
}

in bipolar transistors, ${ }^{16,17}$ it is expected that with exposure to neutrons, the IGBT on-resistance should increase (similar to the power MOSFET), but that the anode current fall time should decrease (not observed in power MOSFETs).

The purpose of this paper is to present results of the effects of neutrons on the characteristics of IGBTs. The results show that the on-state voltage at a given current increases, the switching time decreases, and the saturation current at a given gate voltage decreases as the neutron fluence increases. The experimental results are explained with the aid of an analytic model of the IGBT, ${ }^{18}$ which is briefly reviewed. It is shown that all of the primary effects observed in the IGBT are related to reduction in carrier lifetime and not to changes in material resistivity.

\section{IGBT Device Physics}

The structure of the IGBT is very similar to that of the power MOSFET. A change in conductivity type of one region in the power MOSFET transforms the device into an IGBT. Although structurally very similar, the physics of operation of the two device types is quite different. Most of the power MOSFET characteristics can be understood by modeling the device as a simple MOSFET in series with a resistor (to account for the drain resistance). The IGBT behaves as a bipolar transistor which is supplied base current from a MOSFET and both bipolar and MOSFET device physics must be included in its modeling.

The following discussion is in terms of an n-channel IGBT. There have been some p-channel devices described, but they are not commercially important at this time. First, a physical description of the IGBT structure is given and that structure is related to a relatively simple equivalent circuit for the device. Then, a model for the bipolar transistor portion of the IGBT is presented, emphasizing effects that must be included that are usually unimportant in bipolar transistor modeling. The important mathematical expressions for understanding the effects of neutrons on the IGBT are given. A simple MOSFET model is incorporated with the bipolar transistor model to fully describe the IGBT.

\section{II.a. IGBT Structure}

A schematic cross section of the structure of the IGBT is compared to that of a power MOSFET in figure 1. The only dif-

MOSFET

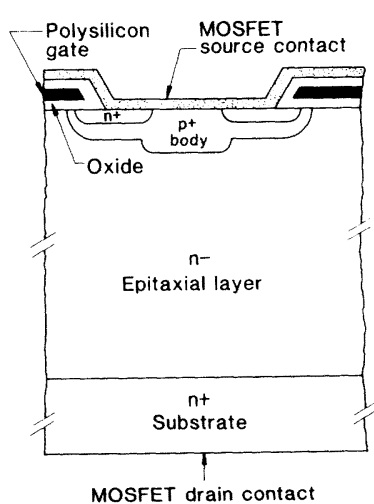

MOSFET drain contact
IGBT

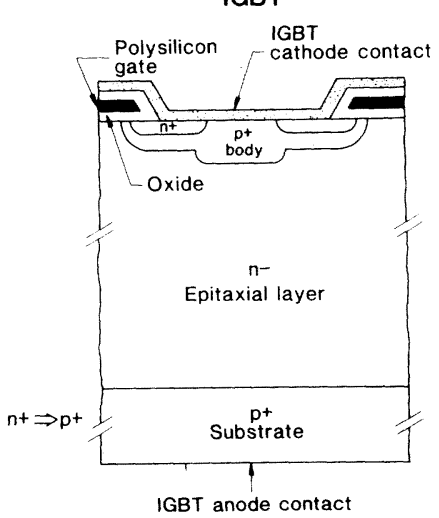

Figure 1. A schematic representation of the IGBT and the power MOSFET structures, indicating that a change of the conductivity type of the substrate transforms the MOSFET into an IGBT. 
ference in the two structures is the replacement of the n-type drain region of the MOSFET with a p-type anode region in the IGBT. The n-type epitaxial region in both devices is thick and has low doping in order to support large anode-cathode (drain-source) voltages. In the power MOSFET, this region contributes to an undesirably large on-resistance when the device is in the on-state. The replacement of the MOSFET ndrain contact by the p-anode in the IGBT serves to create a $\mathrm{p}-\mathrm{n}$ junction (p-substrate $\mathrm{n}$-epitaxial layer). This $\mathrm{p}-\mathrm{n}$ junction acts to reduce the on-resistance in the IGBT on-state by injecting minority carriers into the epitaxial layer, thus reducing its resistivity.

The basic equivalent circuit for the IGBT is shown in figure 2. The IGBT behaves as a bipolar transistor $(p-n-p)$ which is supplied base current (electrons) by an n-channel MOSFET. The p-type anode is the bipolar transistor emitter and the MOSFET body serves as the collector which is shorted to the source of the MOSFET. The source and body diffusions into the epitaxial layer (see figure 1) form MOSFET cells with the channel at the surface of the body and with the portion of the epitaxial layer between the body diffusions serving as the MOSFET drain. There are typically several thousand of these cells per chip, and the MOSFET in the equivalent circuit is a parallel combination of these cells.

The substrate-epitaxial layer (bipolar emitter-base) junction is forward biased when the IGBT is in the on-state, and holes are injected into the epitaxial layer (bipolar transistor base) from the substrate (bipolar transistor emitter). These holes drift and diffuse through the base; some recombine with electrons from the MOSFET and the remainder reach the epitaxial layer-body (bipolar transistor base-collector) junction which is reverse biased by the drain voltage of the MOSFET. These remaining holes are swept into the collector and make up the bipolar transistor collector current.

\section{II.b. Bipolar Transistor Model}

Because of the IGBT structure, the bipolar transistor base current is introduced from the collector end of the base. In the model, the base contact is coincident with the base edge of the base-collector junction, and the base current (electrons) entering this region is equal to the MOSFET channel current. The holes entering this region are swept into the collector and are equal to the collector current. For an optimized IGBT in practical operation, the bipolar transistor has a very low gain $(\sim 1)$, and the base region is operating under high carrier level injection. Because of these conditions, the base region of the bipolar transistor is treated like the intrinsic region of a $p-i-n$ diode but with different boundary conditions. The difference in boundary conditions is that the carrier densities are zero at the collector end of the bipolar transistor base.

The model assumes the equivalent circuit of figure 2 and treats the IGBT in one dimension. A one-dimensional model is adequate because the lateral separation of the MOSFET drains is typically much less than the bipolar transistor base width. As mentioned above, the bipolar transistor base is assumed to be operating under high level injection conditions.

Steady State. At steady state, the excess carrier distribution in the base is given by:

$$
\delta p(x)=P_{0} \frac{\sinh \left(\frac{W-x}{L}\right)}{\sinh \left(\frac{W}{L}\right)}
$$

and the base and collector currents are given by:

$$
I_{B}=\frac{P_{0}^{2} I_{s n e}}{n_{i}^{2}}+\frac{q P_{0} A D}{L}\left(\operatorname{coth}\left(\frac{W}{L}\right)-\frac{1}{\sinh \left(\frac{W}{L}\right)}\right)
$$

$$
I_{C}=\frac{P_{0}^{2} I_{s n e}}{b n_{i}^{2}}+\frac{q P_{0} A D}{L}\left(\frac{\operatorname{coth}\left(\frac{W}{L}\right)}{b}+\frac{1}{\sinh \left(\frac{W}{L}\right)}\right) .
$$

The coordinate system is shown in flgure 3 and the symbols used here are defined in table 1.

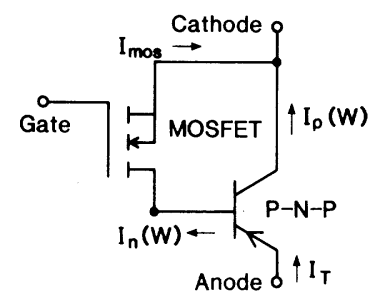

Figure 2. The basic equivalent circuit of the IGBT.

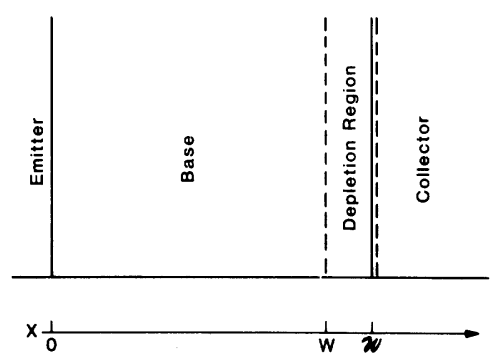

Figure 3. The coordinate system used in deriving expressions for transport in the bipolar transistor base.

\section{TABLE 1}

\section{Symbol Definition}

$\phi_{n}, \phi_{p}$
$V_{g s}$
$V_{e b}$
$V_{A K}$
$\phi_{n}, \phi_{p}$
$I_{B}, I_{C}$
$I_{m o s}=I_{B}$
$I_{s n e}$
$I_{T}=I_{B}+I_{C}$
$I_{T}^{s a t}$
$I_{m o s}^{s a t}$
$n, p$
$\delta p$
$P_{0}$
$Q(t)$
$N_{B}$
$W_{B}$
$A$
$\tau$
$\tau_{H L}$
$n_{i}$
$\mu_{n}, \mu_{p}$
$b$
$D_{n}, D_{p}$
$D=\frac{2 D_{n} D_{p}}{D_{n}+D_{p}}$
$L=\sqrt{D \tau_{H} L}$
$q$
$K$
$k$
$K_{p}$
$V_{T}$
$R_{m o s}$

electron, hole quasi-fermi potential $(V)$ MOSFET gate-source voltage $(V)$ bipolar emitter-base voltage $(V)$ IGBT anode-cathode voltage $(V)$ electron, hole quasi-fermi potential $(V)$ bipolar base, collector current $(A)$ MOSFET current $(A)$ emitter electron saturation current $(A)$ IGBT anode current $(A)$ IGBT saturation current $(A)$ MOSFET saturation current $(A)$ electron, hole carrier concentration $\left(\mathrm{cm}^{-3}\right)$ excess carrier concentration $\left(\mathrm{cm}^{-3}\right)$ $\delta p$ at emitter edge of the base $\left(\mathrm{cm}^{-3}\right)$ excess base charge during transient $(C)$ doping concentration in base $\left(\mathrm{cm}^{-3}\right)$ quasi-neutral base width $(\mu m)$ active chip area $\left(\mathrm{cm}^{2}\right)$ low level excess carrier lifetime in base $(\mu \varepsilon)$ high level excess carrier lifetime in base $(\mu \theta)$ intrinsic carrier concentration $\left(\mathrm{cm}^{-9}\right)$ electron, hole mobility $\left(\mathrm{cm}^{-2} / \mathrm{Vs}\right)$ $\frac{\mu_{n}}{\mu_{p}}$ electron, hole diffusivity $\left(\mathrm{cm}^{2} / \mathrm{s}\right)$ ambipolar diffusivity in base $\left(\mathrm{cm}^{2} / \mathrm{s}\right)$ ambipolar diffusion length in base $(\mu m)$ electronic charge $\left(1.6 \times 10^{-19} \mathrm{C}\right)$ lifetime neutron damage constant $\left(\mathrm{n} \cdot \mathrm{s} / \mathrm{cm}^{2}\right)$ Boltzmann constant MOSFET transconductance $\left(A / V^{2}\right)$ MOSFET threshold voltage $(V)$ MOSFET linear region resistance $(\Omega)$ 
The anode-to-cathode voltage drop of the IGBT is the sum of the bipolar transistor emitter contact to base contact voltage drop (the base contact is at the collector end of the base) and the MOSFET source-drain voltage:

$$
V_{A K}=V_{e b}+I_{m o s} R_{m o s} .
$$

A band diagram including quasi-fermi potentials through the emitter and base of the bipolar transistor is shown in figure 4. This figure is useful for describing the voltage drop across the bipolar transistor. The bipolar transistor emitter contact to base contact voltage difference includes the voltage drops at the emitter-base junction and the voltage drop through the modulated base. Using quasi-fermi levels, as shown in figure 4, this voltage is given by:

$$
V_{e b}=\left(\phi_{p e j}-\phi_{n e j}\right)+\left(\phi_{n e j}-\phi_{n b}\right)
$$

where:

$$
\phi_{p e j}-\phi_{n e j}=\frac{k T}{q} \ln \left(\frac{P_{0}^{2}}{n_{i}^{2}}\right)
$$

is the electron quasi-fermi potential drop in the emitter, and

$$
\phi_{n e j}-\phi_{n b}=\frac{J_{T} W}{\left(1+\frac{1}{b}\right) \mu_{n} q n_{e f f}}-\frac{\tilde{D}}{\mu_{n}} \ln \left[\frac{P_{0}+N_{B}}{N_{B}}\right]
$$

is the electron quasi-fermi potential drop across the lightly doped (but carrier-modulated) base. The first term on the right of eq $\mathbf{7}$ is related to the drift of carriers across the base, and its magnitude is inversely proportional to the "effective" carrier concentration given by:

$$
\frac{1}{n_{\text {eff }}}=\int_{0}^{W} \frac{\mathrm{d} x}{N_{B}+\delta p(x)}
$$

The second term on the right of eq 7 is related to the diffusion of carriers across the base.

Transient. To turn-off the IGBT, the gate voltage is switched below threshold, which rapidly removes the MOSFET channel current and eliminates the base current supplied to the bipolar transistor. The bipolar transistor collector current falls slowly, though, because the charge stored in the base region must decay. The anode current waveform for the IGBT being turned off with a constant anode-cathode voltage is shown in figure 5. The initial rapid drop is associated with removal of the MOSFET channel current, and the long decay tail is due to the remaining slowly decaying excess carriers in the bipolar transistor base.

The slowly decaying portion of the anode current is shown over several decades of current in figure 6 . The lifetime in the bipolar transistor base here is $7 \mu s$. Over most of the current range, the decay of carriers is dominated by recombination in the bipolar transistor base. This is evident from the large linear portion of the plot. At high currents, the reduced emitter efficiency becomes important, and the decay rate is increased by injection of carriers into the bipolar emitter. At low currents, the carrier lifetime decreases as the base leaves the high injection regime and surface and space charge recombination become important; both effects act to increase the decay rate. Over the intermediate current range where recombination dominates, the high level carrier lifetime can be found from the decay rate by:

$$
\tau_{H L}=-\left(\frac{\mathrm{d} \ln I_{T}}{\mathrm{~d} t}\right)^{-1}
$$

The anode current during the recombination dominated portion of the slow decay phase is given by:

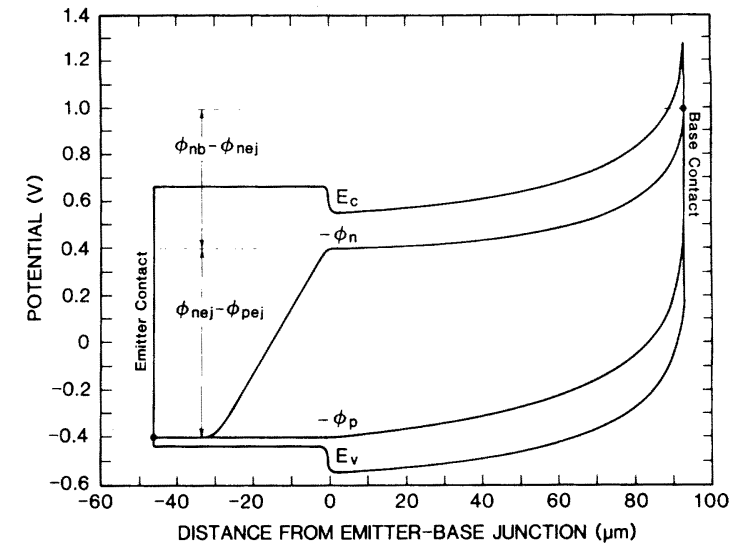

Figure 4. A band diagram from the emitter to base contacts of the bipolar transistor of the IGBT.

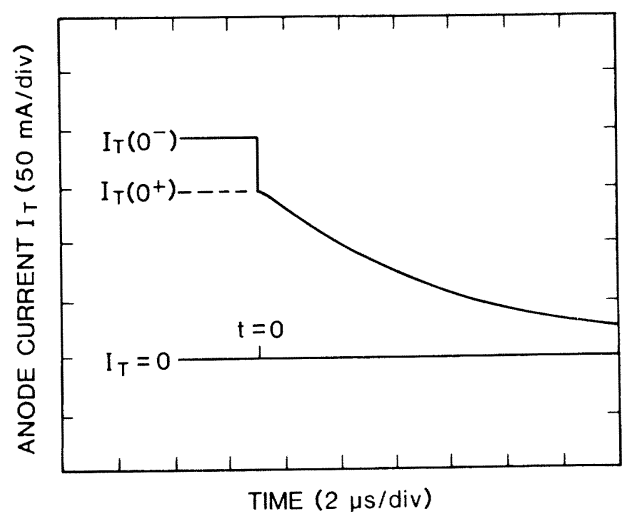

Figure 5. A switching transient current waveform of an IGBT being switched off, which indicates the current immediately before $\left(I_{T}\left(0^{-}\right)\right)$and immediately after $\left(I_{T}\left(0^{+}\right)\right)$the initial rapid fall in current.

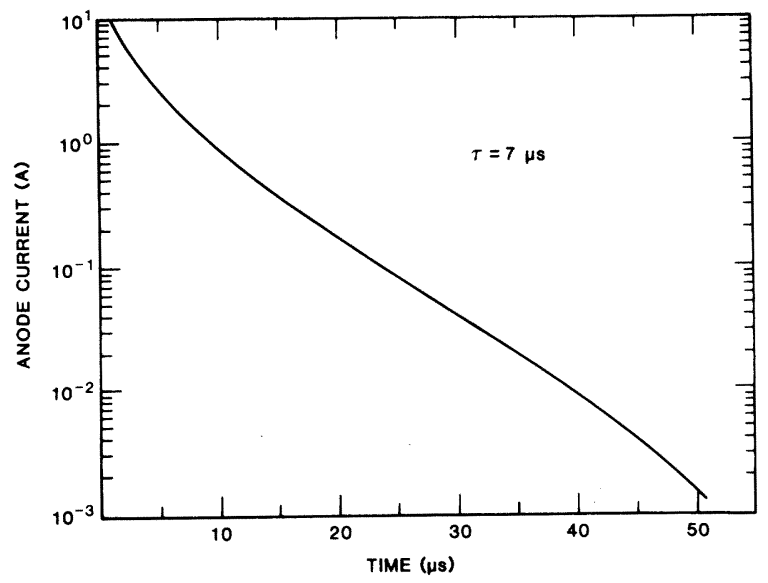

Figure 6. The anode current over several decades of the slowly decaying portion of the switching transient current decay waveform at a constant anode voltage.

$$
I_{T}(t)=\frac{4 D_{p}}{W^{2}} Q(t),
$$

where $Q(t)$ is the total excess carrier charge in the base. The anode current is slightly larger than that given by eq 10 at very high and very low currents. 
The relative magnitude of the rapid drop in current shown in figure 5 to the total steady-state current is closely related to (but not equal to) the current gain of the bipolar transistor. The quantity $\beta_{t r}$ is defined as:

$$
\beta_{t r} \equiv \frac{I_{T}\left(0^{+}\right)}{I_{T}\left(0^{-}\right)-I_{T}\left(0^{+}\right)}
$$

where $I_{T}\left(0^{-}\right)$is the total steady-state current and $I_{T}\left(0^{+}\right)$is the current from which the slow decay begins. The maximum value of $\beta_{t r}$ is:

$$
\beta_{t r, V}^{\max }=\left(\left(\frac{W}{L}\right)^{2} \frac{\operatorname{coth}\left(\frac{W}{L}\right)}{2 \tanh \left(\frac{W}{2 L}\right)}-1\right)^{-1}
$$

The reason that $\beta_{t r}$ is not equal to the bipolar transistor gain is that the component of hole drift current associated with the net electron current at steady state is not present during the slow decay phase.

\section{II.c. MOSFET Model}

For this work, a rather simple model for the MOSFET is sufficient. The resistance of the MOSFET in the linear region is given by:

$$
R_{m o s}=\frac{1}{K_{p}\left(V_{g s}-V_{T}\right)},
$$

where the parameter $K_{p}$ is equal to the product of the oxide capacitance, the surface carrier mobility, and the effective width-to-length ratio of the MOSFET cells. The effect of gate voltage on the surface mobility is neglected. The saturation current is given by:

$$
I_{m o s}^{s a t}=\frac{K_{p}}{2}\left(V_{g s}-V_{T}\right)^{2},
$$

where saturation is assumed to be due to pinch-off.

\section{Experiment}

A group of IGBT devices with similar electrical characteristics was chosen and the devices were irradiated at one of three different neutron fluences. The devices were rated by their manufacturer to have a breakdown voltage of $450 \mathrm{~V}$ and a maximum current of $20 \mathrm{~A}$. The devices were screened to have similar pre-rad values of bipolar transistor base minority carrier lifetime and MOSFET transconductance. The threshold voltages of the devices were not matched as this parameter is unimportant to the study and the account of changes in the characteristics of the IGBT due to threshold voltage shifts can easily be made.

The White Sands Missile Range Fast Burst Reactor was used for the neutron exposures. The devices were exposed to nominal neutron fluences of $10^{11}, 10^{12}$, and $10^{13}$ neutrons $/ \mathrm{cm}^{2}$ $\left(E_{n}>10 \mathrm{keV}\right)$. The fluences were determined from sulfur pellet measurements and a plutonium-sulfur ratio supplied by the facility. Typical levels of accompanying ionizing radiation were inferred from previous work ${ }^{5}$ (e.g., $2.4 \times 10^{3} \operatorname{rad}(\mathrm{Si})$ at $10^{13}$ neutrons $/ \mathrm{cm}^{2}$ ) and their effects are secondary to those of the neutrons.

\section{III.a. Effect of Neutrons on Physical Parameters}

The important physical material parameters that change with exposure to neutrons are the excess carrier lifetime $(\tau)$, the mobility $(\mu)$, and the effective dopant density $\left(N_{B}\right)$. The lifetime and mobility are reduced by the introduction of lattice damage and the associated trapping/recombination centers. The effective dopant density is reduced by the introduction of damage induced deep level compensation centers. Changes in either of these parameters change the characteristics of bipolar transis-

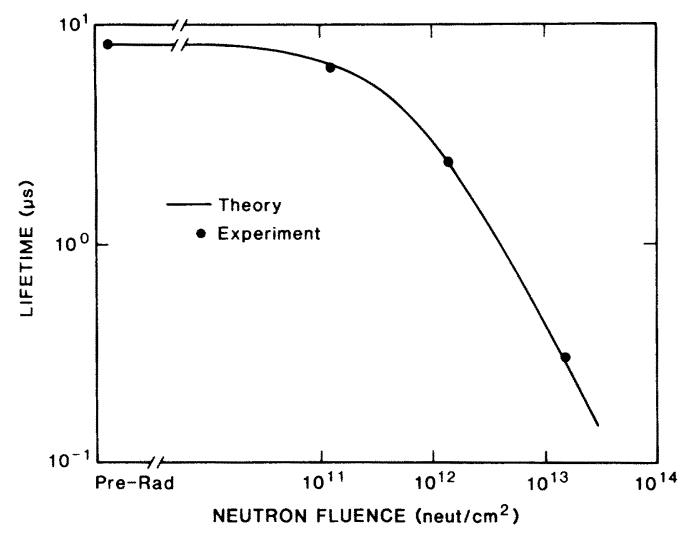

Figure 7. Measured values and a theoretical fit to the empirical expression for lifetime versus neutron fluence.

tors, and so it is expected that the output characteristics of the IGBT change with exposure to neutrons.

The change in effective dopant concentration in the bipolar transistor base is slight $\left(\delta N_{B}<N_{B}\right)$ for the bipolar transistor base dopant densities and range of fluences chosen. ${ }^{8}$ These slight changes have no perceptible effect on the characteristics of the bipolar transistor and thus have no effect on the output characteristics of the IGBT for the fluence range of this experiment.

The principal effect of neutron exposure on the IGBT characteristics is through changes in excess carrier lifetime in the bipolar transistor base region. An empirical expression for lifetime as a function of fluence is: ${ }^{16}$

$$
\frac{1}{r}=\frac{1}{\tau_{0}}+\frac{\phi}{K}
$$

The measured values of high level lifetime fit the expression very well using a value of $K=4.4 \times 10^{6}$ neutrons $\cdot \mathrm{s} / \mathrm{cm}^{2}$. The measured and calculated values of the high level lifetime are shown in figure 7 . The high level lifetimes were measured from the exponentially decaying portion of the turn-off current waveform, such as shown in figure 5 . These measurements were made in the intermediate current range discussed for figure 6 where the base is in high level injection and injection into the emitter does not significantly affect the decay rate.

\section{III.b. Effect of Neutrons on IGBT Electrical Characteristics}

The measured and predicted on-state voltage versus current are shown in figure 8 for each of the neutron fluence levels. These data are replotted in figure 9 as on-state voltage versus neutron fluence for several values of current. The effect of increasing neutron fluence is to increase the on-state voltage for a given current level. The predicted curves are found using eq 1 through eq 8 and eq 13. The material parameters and lifetimes used for these calculations are listed in tables 2 and 3.

The relative magnitude of the slowly decaying portion of the turn-off current waveform compared to the steady-state current, i.e., $\beta_{t r}$ from eq 11, was measured for all the devices. The measurements were made as a function of current. Examples of the turn-off waveform after each fluence level are shown in figure 10 , and the measured and predicted values for $\beta_{t r}$ are shown in figure 11. The slowly decaying portion of the current turn-off waveform is decreased in initial magnitude and the decay time becomes shorter with increasing neutron fluence. 


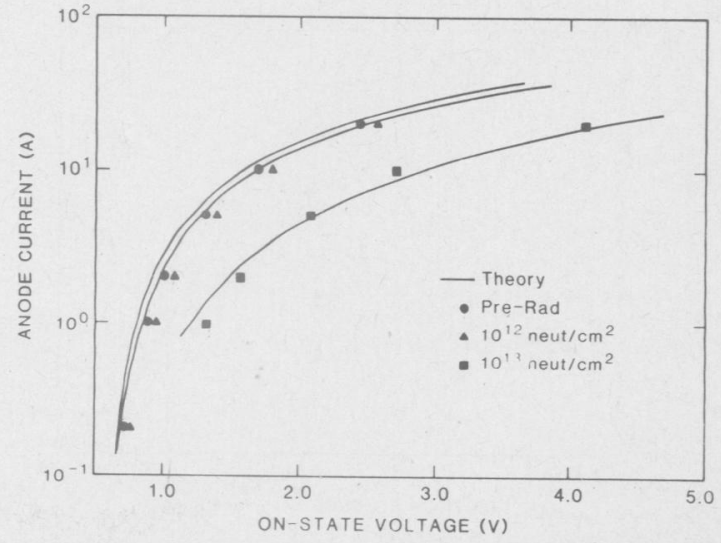

Figure 8. Predicted and measured on-state voltage versus current for the devices before and after neutron exposure.

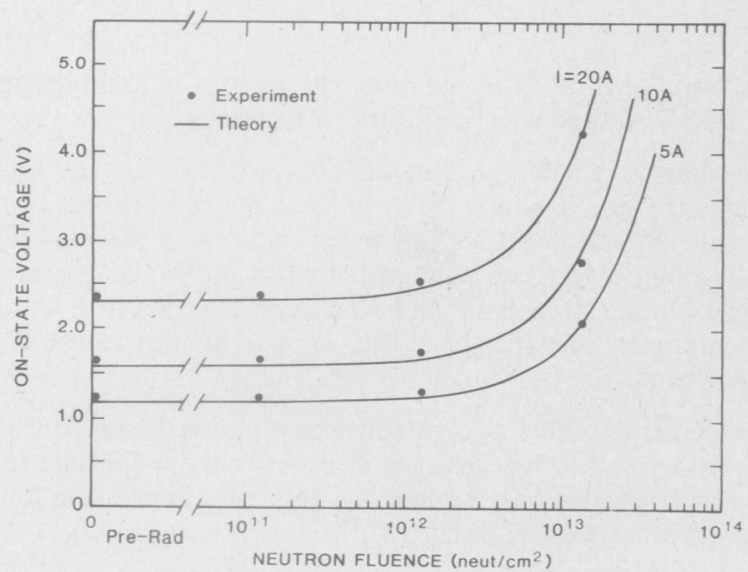

Figure 9. The predicted and measured variation of the on-state voltage at a given current with neutron fluence.

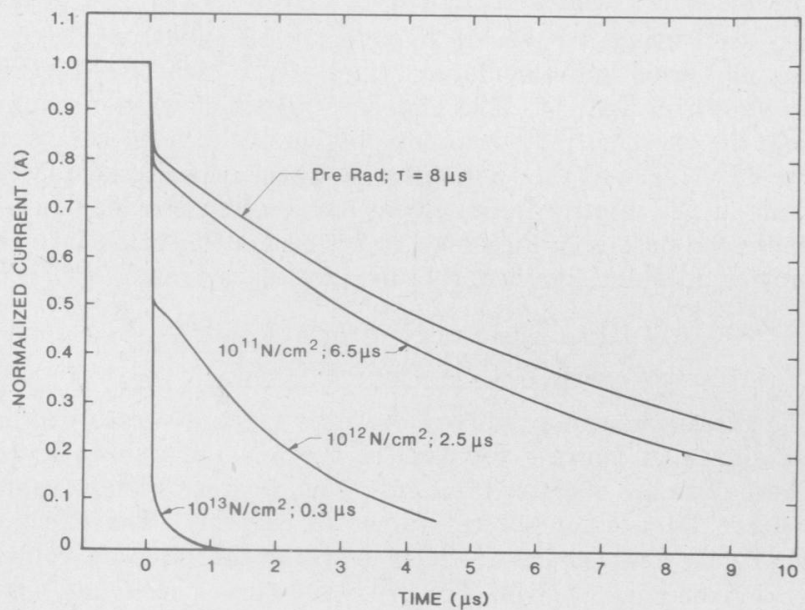

Figure 10. Turn-off switching waveforms of the IGBTs before irradiation and at the various neutron fluences of this experiment, indicating the measured exponential current decay rate of the slowly decaying tail.

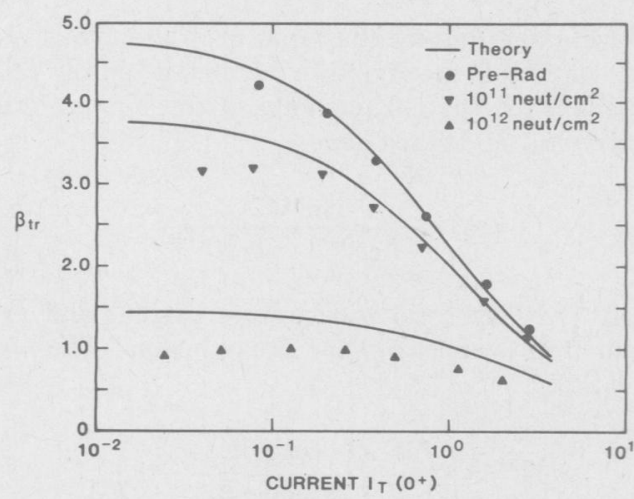

Figure 11. The measured and predicted relative initial size of the slowly decaying current tail to the value of steady-state current, as a function of current for the IGBTs before irradiation and at various neutron fluences.

TABLE 2

$\begin{array}{ll}\text { Neutron Fluence } & \text { High Level Lifetimes } \\ \text { Pre-Rad } & 8.1 \mu s \\ 10^{11} & 6.45 \mu s \\ 10^{12} & 2.45 \mu s \\ 10^{13} & 0.3 \mu s\end{array}$

TABLE 3

Device Parameters

$\begin{array}{ll}N_{B} & 2 \times 10^{14} \mathrm{~cm}^{-3} \\ W_{B} & 93 \mu \mathrm{m} \\ I_{s n e} & 7 \times 10^{-14} \mathrm{~A} \\ K_{p} & 0.36 \mathrm{~A} / \mathrm{V}^{2} \\ A & 0.1 \mathrm{~cm}^{2} \\ \mu_{p} & 450 \mathrm{~cm}^{2} / \mathrm{Vs} \\ \mu_{n} & 1500 \mathrm{~cm}^{2} / V \\ n_{i} & 1.45 \times 10^{10} \mathrm{~cm}^{-3}\end{array}$

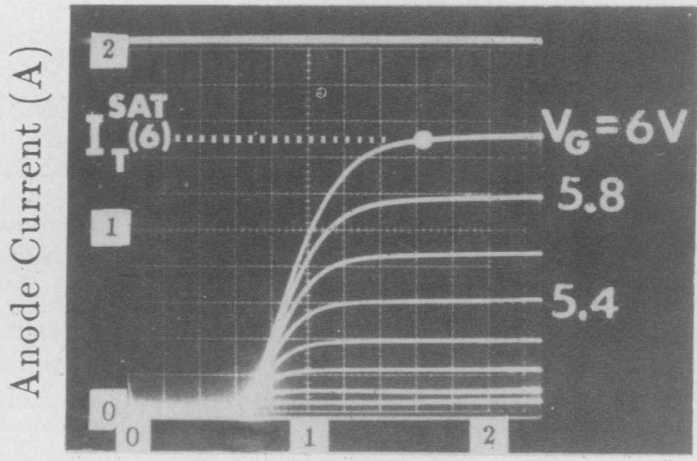

Anode Voltage (V)

Figure 12. An oscillogram of the steady-state characteristics of an IGBT, defining the value of the IGBT saturation current at a given gate voltage. 
The output characteristics for an IGBT as measured on a curve tracer are shown in figure 12. On this figure, the IGBT saturation current at a given gate voltage, $I_{T}^{s a t}$, is defined as the current above which increases in the anode voltage have no effect on the current. Saturation occurs in the IGBT because the MOSFET which is supplying base current to the bipolar transistor saturates. The MOSFET saturation current is given by eq 14 . The measured and predicted values of the IGBT saturation current after each neutron fluence versus gate voltage are shown in figure 13. The saturation current decreases as the neutron fluence increases. The predictions are made by multiplying the MOSFET current by the gain enhancement of the bipolar transistor:

$$
I_{T}^{s a t}=I_{m o s}^{s a t}\left(\frac{I_{C}+I_{B}}{I_{B}}\right)
$$

where $I_{C}$ and $I_{B}$ are calculated from eq 2 and eq 3.

\section{Discussion}

The excess carrier concentrations as a function of distance from the emitter end to the collector end of the base of the bipolar transistor for two different lifetimes and the same current density are shown in figure 14. These carrier distributions are calculated from eq 1 . The most important feature to note is that as the lifetime is decreased, the excess stored charge decreases every where in the base. The relative lifetimes chosen for these plots are approximately those for an unirradiated IGBT and for a device after a dose of between $10^{12}$ and $10^{13}$ neutrons $/ \mathrm{cm}^{2}$. The major effects that neutrons have on the electrical characteristics of the IGBT can be qualitatively described with the aid of this figure.

Consider first the increase in on-state voltage at a given current as the neutron fluence increases, such as shown in figures 8 and 9. From eq 8 , it can be seen that as the excess carrier concentration in the base decreases, $n_{\text {eff }}$ decreases. This causes an increase in the electron quasi-fermi potential drop across the base, as given by eq 7 . In effect, as less charge is stored in the base, its effective resistance increases, contributing to an increase in the on-state voltage.

Consider next the decreased magnitude and decay time of the slowly decaying portion of the turn-off current waveform, as shown in figures 10 and 11 . The magnitude of the current during the slow decay phase is given by eq 10 . From eq 10 , it can be seen that: 1) the smaller the magnitude of stored charge, the smaller the current; and 2) the more rapid the decay of stored charge, the more rapid the decay in current. The decay time of the stored charge is shorter for the lower lifetime device because the recombination rate of excess carriers is greater. The initial magnitude of the slowly decaying tail is smaller for the lower lifetime device because the initial stored charge is less.

The saturation current of the IGBT decreases with increasing neutron fluence because as the lifetime of the bipolar transistor base is decreased, the gain of the bipolar transistor is reduced. Because the value of the MOSFET saturation current, given by eq 14, does not change with dose, the IGBT saturation current is decreased by the ratio of decrease in the bipolar transistor current gain, given in eq 16 . Because the current gain of the reduced lifetime bipolar transistor is reduced, a given value of MOSFET saturation current (bipolar transistor base current) results in a lower value of bipolar transistor collector current and thus a lower value of IGBT saturation current.

It is of interest to compare the effects of neutrons on the IGBT with their effects on power MOSFETs. It has been shown that the on-resistance of power MOSFETs increase with increasing neutron fluence. ${ }^{5}$ This effect in the power MOSFET is due to

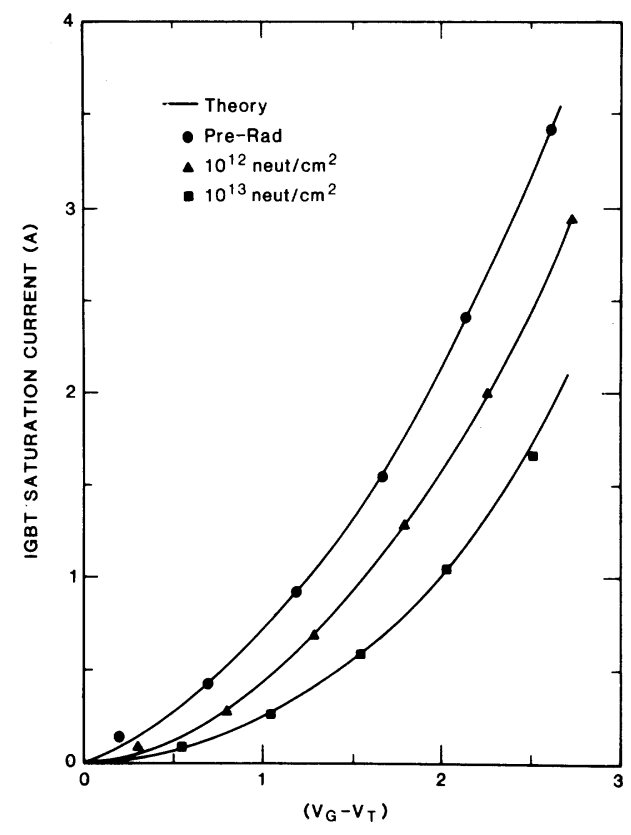

Figure 13. The measured and predicted values of the IGBT saturation current versus gate voltage for the IGBTs before irradiation and at various neutron fluences.

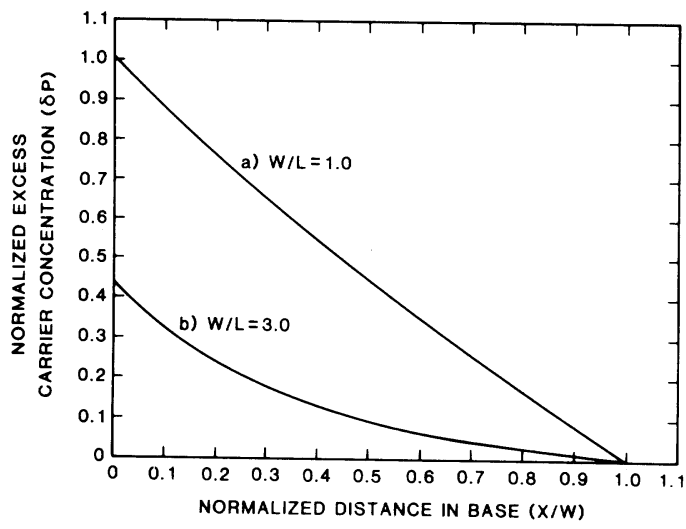

Figure 14. A schematic representation of the excess carrier distribution from the emitter to collector ends of the bipolar transistor base of an IGBT before irradiation $(W / L \approx 1.0)$ and after exposure to a neutron fluence of between $10^{12}$ and $10^{13}$ neut $/ \mathrm{cm}^{2}(W / L=3.0)$, both at a given current.

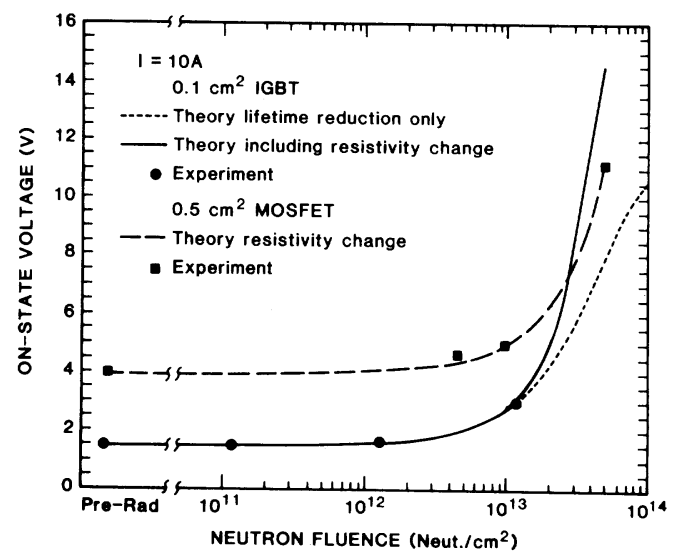

Figure 15. A comparison of the variation of the on-state voltage with neutron fluence between the $0.1 \mathrm{~cm}^{2}$ IGBT and a large area $\left(0.5 \mathrm{~cm}^{2}\right)$ MOSFET. 
a decrease in the effective dopant concentration in the drain of the device. Figure 15 shows a comparison of the effects of neutrons on the on-state voltage between an IGBT (see figure 9 ) and a power MOSFET. The curves in the figure are for a current of $10 \mathrm{~A}$ in each instance. The effect of the neutrons on the MOSFET are based upon the results shown in figure 5 (device A) of Blackburn, et al. ${ }^{5}$ The pre-rad MOSFET drainsource on-resistance is assumed to be $0.4 \Omega$. Although the two devices compared are not totally equivalent, the relative trends are clear. The on-state voltage of the IGBT begins to increase at a lower neutron fluence than for the power MOSFET. This is because, as previously mentioned, the lifetime begins to decrease at a lower fluence than does the effective doping density. Included in the IGBT extrapolated results (solid curve of figure 15 ) is the predicted effect of the reduction in effective doping on that device. ${ }^{8}$ At high doses, the IGBT characteristics become those of a power MOSFET, which has an area of 0.2 times that of the 0.4 $\Omega$ MOSFET.

Unlike the IGBT, the power MOSFET does not experience any change in switching characteristics with increasing neutron fluence. This is because the switching of the power MOSFET is primarily controlled by the gate and drain capacitances, both of which are unaffected by neutrons. Also, unlike the IGBT, the power MOSFET experiences no change in saturation current at a given gate voltage with increasing neutron fluence.

\section{Conclusions}

Because the output characteristics of the IGBT are dominated by the wide-base bipolar transistor, the IGBT output is very sensitive to the carrier lifetime in the base of the bipolar transistor. The bipolar transistor base lifetime is found directly from the exponential decay of the tail portion of the anode current waveform. The measured lifetimes decrease with neutron exposure as expected.

The on-state voltage at a given current increases, the switching time decreases, and the saturation current at a given gate voltage decreases as the neutron fluence increases. The experimental results can be explained with the aid of an analytic model of the IGBT where the carrier lifetime is reduced by the neutron exposure. The effects of neutrons on the IGBT are related to reduction in carrier lifetime and not to changes in material resistivity.

Both the increased on-state resistance and the decreased total turn-off time must be considered for an IGBT which experiences neutron radiation. The increased on-resistance, of course, contributes to increased on-state power dissipation within the IGBT and increased operating temperature. The low on resistance is the principal advantage of the IGBT over the MOSFET and this advantage is lost at high neutron doses. The decreased turn-off time may reduce power dissipation during switching. Assuming that the unirradiated IGBT was optimized to the circuit, the sum of the on-state and switching power dissipation would likely increase.

\section{Acknowledgments}

The authors wish to thank Tom Ellis and Young Kim of the Naval Weapons Support Center, Crane, Indiana, for providing the neutron exposures at the White Sands Missile Range.

\section{References}

1. J. P. Russell, A. M. Goodman, L. A. Goodman, and J. M. Neilson, "The COMFET-A New High Conductance MOS-Gated Device," IEEE Electron Device Lett. EDL-4, $63(1983)$
2. B. J. Baliga, M. S. Adler, R. P. Love, P. V. Gray, and N. D. Zommer, "The Insulated Gate Transistor: A New Three Terminal MOS-Controlled Bipolar Power Device," IEEE Trans. Electron Devices 31, 828 (1984).

3. W. E. Baker, Jr., "The Effect of Radiation on the Characteristics of Power MOSFETs," Proc. POWERCON 7, D3-1 (1980).

4. S. Rattner, "Additional Power VMOS Radiation Effects Studies," IEEE Trans. Nucl. Sci. NS-27, 1329 (1980).

5. D. L. Blackburn, T. C. Robbins, and K. F. Galloway, "VDMOS Power Transistor Drain-Source Resistance Radiation Dependence," IEEE Trans. Nucl. Sci. NS-28, 4380 (1981).

6. W. E. Abare and W. K. Martindale, "Dose Rate Tolerant HEXFET Power Supply," IEEE Trans. Nucl. Sci. NS-29, 4380 (1982).

7. S. S. Seehra and W. J. Slusark, Jr., "The Effects of Operating Conditions on the Radiation Resistance of VDMOS Power FET's," IEEE Trans. Nucl. Sci. NS-29, 1559 (1982).

8. H. Volmerange and A. A. Witteles, "Radiation Effects on MOS Power Transistors," IEEE Trans. Nucl. Sci. NS-29, 1565 (1982).

9. D. L. Blackburn, D. W. Berning, J. M. Benedetto, and K. F. Galloway, "Ionizing Radiation Effects on Power MOSFETs During High Speed Switching," IEEE Trans. Nucl. Sci. NS-29, 1555 (1982).

10. D. L. Blackburn, J. M. Benedetto, and K. F. Galloway, "The Effect of Ionizing Radiation on the Breakdown Voltage of Power MOSFETS," IEEE Trans. Nucl. Sci. NS-30, 4116 (1983)

11. D. M. Jobson-Scott, "An Investigation into Radiation Induced Second Breakdown in N Channel Power MOSFETs," IEEE Trans. Nucl. Sci. NS-31, 1508 (1984).

12. D. M. Jobson-Scott and G. B. Roper, "Neutron Radiation as a Means of Reducing the Incidence of Radiation Induced Breakdown in a Radiation Hard Power MOSFET," IEEE Trans. Nucl. Sci. NS-32, 3961 (1985).

13. A. Mogro-Camper, R. P. Love, M. F. Chang, and R. F. Dryer, "Shorter Turn-Off Times in Insulated Gate Transistors By Proton Implantation," IEEE Electron Device Lett. EDL-6, 224 (1985).

14. W. A. Strifler and B. J. Baliga, "Comparison of Neutron and Electron Irradiation for Controlling IGT Switching Speed," IEEE Trans. Electron Devices ED-32, 1629 (1985).

15. H. Yilmaz, W. R. Van Dell, K. Owyang, and M. F. Chang, "Insulated Gate Transistor Modeling and Optimization," IEDM Tech. Dig. 274 (1984).

16. J. R. Srour, "Stable-Damage Comparisons for NeutronIrradiated Silicon," IEEE Trans. Nucl. Sci. NS-20, 190 (1973).

17. M. S. Cooper, J. P. Retzler, and G. C. Messenger, "Combined Neutron and Thermal Effects on Bipolar Transistor Gain," IEEE Trans. Nucl Sci. NS-26, 4758 (1979).

18. A. R. Hefner and D. L. Blackburn, "Performance TradeOff for the Insulated Gate Bipolar Transistor: Buffer Layer versus Base Lifetime Reduction," PESC '86 Record, IEEE Power Electronics Specialists Conference 1986, pp 27-38. 